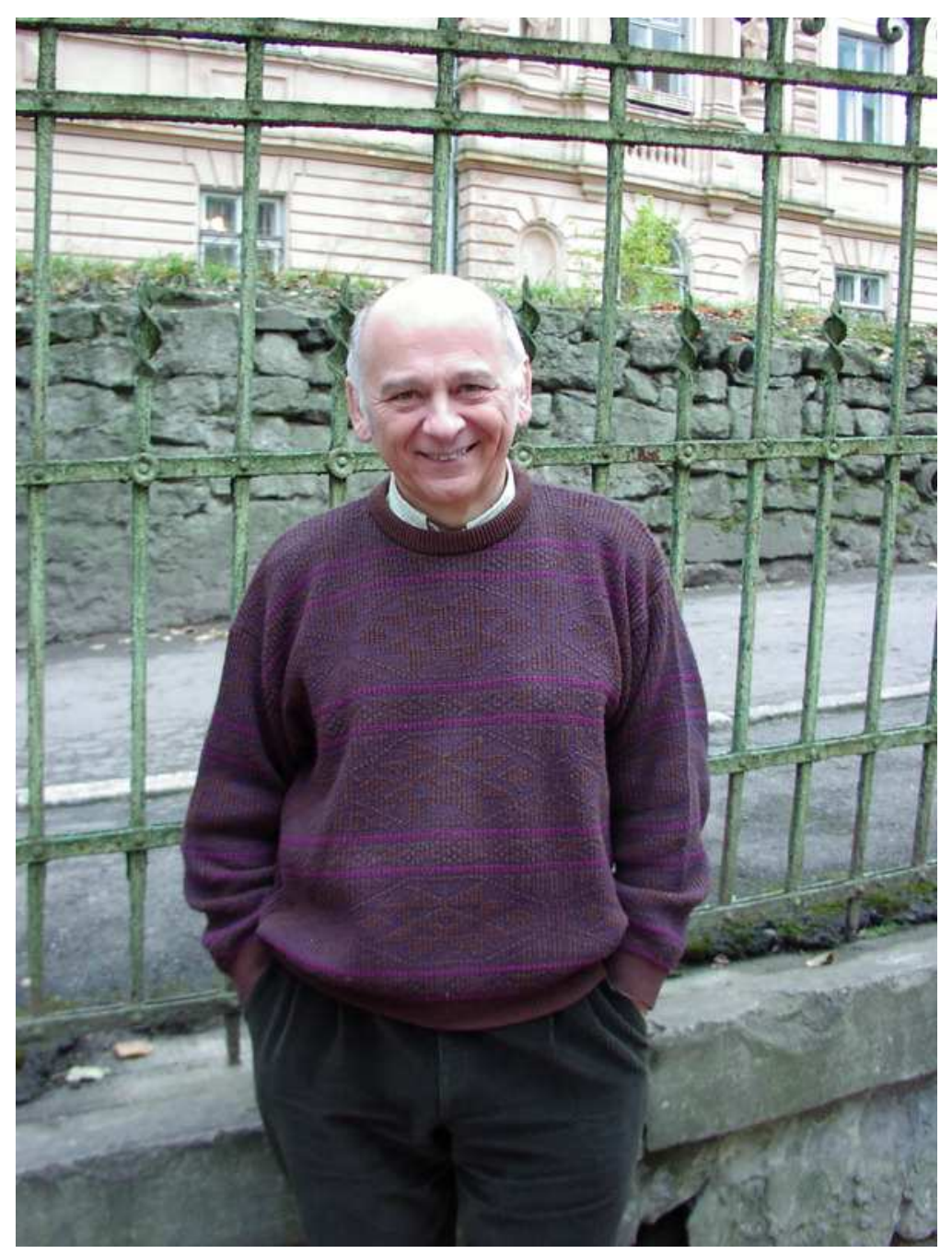

\title{
Main points in my career
}

I started my scientific life with theoretical and experimental works concerning the dielectric relaxation of ionic solutions. After that a large part of my scientific life will be devoted to the theory of charged and polar systems. The investigations on ionic systems suggest to study liquid metals as another example of charged medium. When a metal and an ionic solution are put together they form an electrochemical interface. In the early 1980's we proposed the first calculations showing explicitly the role of electronic distribution in the electric properties of an ideally polarizable electrode. In parallel, the effect of the image potential on the structure of the interface has been investigated and some exact results have been obtained by summation of diagrammatic expansions.

In order to investigate the basic properties of a charged interface (entropy, competition between electrostatic and non-electrostatic interaction potential, for instance) we have developed an approach based on the methods of field theory. This approach has been recently used to describe the fluctuations of charges near a neutral hard 
wall. Going beyond the mean field approximation exact results have been obtained with a simple model. They clearly illustrate the difference between a field theoretic approach and the density functional theory. We have described a new type of phase transition (the desorption transition) in terms of Lie group properties.

As far as the ideally polarizable interface represents the simplest system in Electrochemistry, it was tempting to investigate even the simplest systems like liquid gas-interface or much more complicated ones like those which appear in some technological devices. The two directions have been tried.

The formation of a passive layer on a metal surface is a general problem which has strong practical consequences on the performance of electrical power sources. We have shown that this problem of technological interest leads to very fundamental questions in physics, such as : self organized criticality, chaotic behaviour and quasicyclic processes. All these processes may play a crucial role in our environment.

In the case of simple interface we have investigated the role of the adsorbateadsorbate interactions in the structure of the interface especially when the substrate is not infinitely rigid. An illustration herein is the effect of adsorbate-adsorbate interactions on the roughening transition and the formation of a new structure in the substrate. A natural extension of this research is to investigate the insertion of ions into a deformable host material. More generally, this suggests us to study some systems where we have a coupling between electrical and mechanical properties. Such a coupling appears in the ion insertion processes, in the case of hydrogen sorption in pure metal or alloys and also in the charging/discharging processes developed in polymers grafted on a metallic surface. This represents a new field of investigations to which an anomalous diffusion and possibly non-standard thermodynamics are associated.

During his life a theoretician has to deal with very fundamental problems. Recently I tried to investigate a problem proposed by R.Feynman. Can we derive the path integral formalism in statistical physics directly from the motion of particles? I have shown that this problem leads to very fundamental questions such as: what is the meaning of quantum paths in statistical physics (real processes or just a mathematical trick), what is the status of fractals in quantum theory (a useful mathematical trick or an unavoidable ingredient in the laws of the Nature), how to combine restricted relativity theory with the fractal paths on which the velocity is undefined for a short time interval, does there exist a fractal time in parallel to the fractal character of quantum paths,... . All these questions give us clear goals for the next few years.

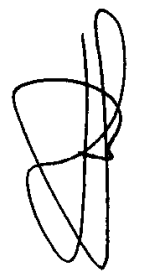

Jean-Pierre Badiali 


\section{Main publications}

1. Lestrade J.C., Badiali J.P., Cachet H. Dielectric relaxation process in electrolyte solutions. - In: Dielectric and Related Molecular Processes. Editor Davies M. London, The Chemical Society, 1974.

2. Badiali J.P., Rosinberg M.L., Goodsiman J. Effect of solvent on the properties of the liquid metal surface. // J. Electroanal. Chem., 1981, vol. 131, p. 130.

3. Badiali J.P., Rosinberg M.L., Goodsiman J. Contribution of the metal to the differential capacity of an ideally polarizable electrode. // J. Electroanal. Chem., 1983, vol. 143, p. 73.

4. Badiali J.P. Structure of the dipolar hard sphere near a neutral hard wall: the orientational structure. // Mol. Phys., 1985, vol. 55, p. 939.

5. Amokrane S., Badiali J.P. A new analysis of the differential capacitance of an ideally polarizable electrode. // J. Electroanal. Chem., 1989, vol. 266, p. 21.

6. Badiali J.P. Structure of a polar fluid near a wall. Exact asymptotic behaviour of the profile, relation with the electrostriction phenomena and the Kerr effect. // J. Chem. Phys., 1989, vol. 90, p. 4401.

7. Zhang Q., Badiali J.P. Calculation of the critical exponents by a renormalization of the Ornstein-Zernike equation. // Phys. Rev. Letters, 1991, vol. 67, p. 1598.

8. Zhang Q., Badiali J.P. Critical phenomena in liquid phases: renormalization from the Ornstein-Zernike equation. // Phys. Rev. A, 1992, vol. 45, p. 8666.

9. Amokrane S., Badiali J.P. Analysis of the capacitance of the metal-solution: role of the metal and metal-solvent coupling. // Modern Aspects of Electrochemistry, 1992, vol. 22, p. 1.

10. Holovko M.F., Badiali J.P. Effect of an association potential on percolation. Applications to reverse micelles containing proteins. // Chem. Phys. Lett., 1993, vol. 204, p. 511.

11. Nainville I., Lemarchand A., Badiali J.P. Growth dynamics and morphology of passive films. // Phys. Rev. E, 1996, vol. 53, p. 2537.

12. Cassin G., Duda Y., Holovko M., Badiali J.P., Pileni M.P. Cytochrome-c in AOT reverse micelles. SAXS measurements, percolation process and critical behaviour. An interpretation in terms of association model. // J. Chem. Phys., 1996.

13. Vorotyntsev M.A., Badiali J.P., Viel E. Multi-component diffusion approach to transport across electroactive-polymer films with two mobile charge carriers. // Electrochimica Acta, 1996, vol. 41, p. 2313. 
14. di Caprio D., Badiali J.P. Invariance properties and correlation function in liquid state theory. Connection with the Ward-Takahashi identities. // Phys. Rev. E, 1996, vol. 53, p. 2537.

15. Lafage M., Windel D., Russier V., Badiali J.P. Mechanism of growth and corrosion at the lithium solvent interface. // Electrochimica Acta, 1997, vol. 42, p. 2841.

16. Stafiej J., Ekoka A., Borkowska Z., Badiali J.P. A simple description of the structure of ionic solutions at electrified interfaces. // Condens. Matter Phys., 1997, No. 12, p. 51-62.

17. Vakarin E.V., Filippov A., Badiali J.P. Distortion of a substrate by adsorption at solid-liquid interface. // Phys. Rev. Lett., 1998, vol. 81, p. 3904.

18. Pecina O., Badiali J.P. Influence of a modulated surface on the properties of liquid-liquid interfaces. // Phys. Rev. E, 1998, vol. 58, p. 6041.

19. Vakarin E., Filippov A., Badiali J.P., Holovko M. Structural rearrangement of solid surfaces due to the competing adsorbate substrate interactions. // Phys. Rev. E, 1999, vol. 60, p. 660.

20. Stafiej J., Badiali J.P. Double layer theory. A new point of view. - In: Computational Methods in Colloid and Interfacial Science. Marcel Dekker, 1999.

21. Badiali J.P. Fractal behaviour in quantum statistical physics. // Phys. Rev. E, 1999, vol. 60, p. 2533.

22. Stafiej J., di Caprio D., Badiali J.P. Desorption transition at charged interfaces. Theoretical approach and experimental evidence. // Phys. Rev. E, 2000, vol. 61 , p. 3877 .

23. di Caprio D., Filippov A., Stafiej J., Badiali J.P. Bicontinuous phases in coulombic systems. The role of specific interactions. // J. Mol. Liquids, 2000, vol. 87, p. 163.

24. Badiali J.P. Fractal behaviour of quantum paths in statistical physics. // Condens. Matter Phys., 2000, vol. 3, No. 3(23), p. 545-558.

25. Vakarin E.V., Badiali J.P., Levi M.D., Aurbach D. Role of the host distortion in the intercalation process. // Phys. Rev. B, 2001, vol. 63, p. 014304.

26. Vakarin E.V., Holovko M.F., Badiali J.P. Adsorbate-induced distortion of solid surfaces. Application to $\mathrm{HCl}$ on ice at stratospheric conditions. // Condens. Matter Phys., 2001, vol. 4, No. 2(26), p. 251-260. 